DOI: $10.35381 /$ racji.v4i7.368

El derecho público como regulador de las relaciones entre las personas

Public law as a regulator of relations between people

\author{
Edison Aroldo Gracia Panta \\ edigrapa@hotmail.com \\ Universidad Laica Eloy Alfaro de Manabí, Manta \\ Ecuador \\ https://orcid.org/0000-0001-8271-9139 \\ Liliana Hipatía Rodríguez Mera \\ hipati710321@yahoo.com \\ Universidad Laica Eloy Alfaro de Manabí, Manta \\ Ecuador \\ https://orcid.org/0000-0003-4087-4362 \\ Klevis Gabriel Gracia Panta \\ Klevisgracia472@hotmail.com \\ Universidad Laica Eloy Alfaro de Manabí, Manta \\ Ecuador \\ https://orcid.org/0000-0002-1407-3812
}

Recibido: 23 de mayo de 2019

Aprobado: 15 de junio de 2019

\title{
RESUMEN
}

El derecho público permite a la ciudadanía acceder a los procesos de justicia en los cuales se ve inmersa desde una postura gratuita, diferenciándose del derecho privado, por lo tanto se tuvo como objetivo analizar el derecho público como regulador de las relaciones entre las personas. Lo cual permitió realizar un análisis estadístico para describir el comportamiento del derecho público y las relaciones personales de quienes se ven envueltos en el proceso judicial, siendo este concebido como servicio público el cual debe ser ofrecido en calidad al cliente. se evidencia como consecuencia que las personas encuestadas manifiestan inconformidad con el servicio prestado a la luz del trato generado por los servidores relacionados al derecho público, producto de un abordaje personal donde no se considera la empatía como una competencia emocional que debe ser abordada para la consideración del establecimiento de una interrelación basada en el respeto mutuo. 
Descriptores: Derecho administrativo; Derecho público; Sistema jurídico; Deontología.

\begin{abstract}
Public law allows citizens to access the justice processes in which it is immersed from a free position, differentiating itself from private law, therefore it was aimed at analyzing public law as a regulator of relations between people. This allowed a statistical analysis to describe the behavior of public law and the personal relationships of those who are involved in the judicial process, this being conceived as a public service which must be offered as a client. It is evidenced as a consequence that the respondents express disagreement with the service provided in the light of the treatment generated by the servers related to public law, product of a personal approach where empathy is not considered as an emotional competence that must be addressed for consideration of establishing an interrelation based on mutual respect.
\end{abstract}

Descriptors: Administrative law; Public law; Legal systems; Deontology.

\title{
INTRODUCCIÓN
}

El presente tema hace una revisión al derecho público y las relaciones entre las personas que se encuentran inmersas en el proceso judicial, de ese modo, se hace necesario acudir a Aristóteles y su postura sobre la teoría de la causalidad, la cual permite partir que todo hecho posee una causa y consecuencia, generándose la interrelación entre dos objetos o fenómenos que interactúan para confluir en acciones positivas o negativas, según sea la causa de origen y consecuencia (repuesta), así se articula el análisis del derecho público y las interrelaciones sociales desde las siguientes perspectivas:

\section{Derecho público}

El derecho público es una rama que permite a la ciudadanía acceder a los procesos de justicia en los cuales se ve inmersa desde una postura gratuita, diferenciándose del derecho privado, así existen funciones que las personas encargadas de cumplir el rol de servidores públicos, no pueden ejercer o ejecutar en el derecho público, limitándose al cumplimiento de lo expresado en la norma. Así Borja Cevallos (2019), destaca que: 
- El Derecho Público regula las relaciones en que interviene el Estado como ente político soberano, dotado de jus imperii sobre la sociedad, mientras que el Derecho privado lo hace cuando comparece a un acto con su personalidad jurídica privada.

- El Derecho Público regula las relaciones en que interviene el Estado como ente político soberano, dotado de jus imperii sobre la sociedad, mientras que el Derecho privado lo hace cuando comparece a un acto con su personalidad jurídica privada.

- En el ámbito del Derecho público prevalece el principio de que los órganos del poder no pueden hacer algo para lo que no estén expresamente autorizados por la ley, mientras que en el del Derecho privado rige el principio contrario: las personas pueden hacer todo lo que no les esté prohibido.

El Estado se ve representado por el derecho público en doble entrada, la primera porque como ente supremo hace cumplir con las leyes, en segundo porque brinda seguridad y asistencia a quienes no tienen la posibilidad de acudir al derecho privado. Esto genera una interrelación entre las personas que puede ser favorable o no, según el servicio procesado, quedando a las expectativas de los usuarios, el valorar el derecho público ecuatoriano.

En este sentido, el derecho público debe ser provisto como un enfoque de servicio de calidad al cliente, Parasuraman, Zeithaml y Berry (2005), señalan que la calidad al servicio se puede medir desde elementos tangibles, fiabilidad, capacidad de respuesta, seguridad (credibilidad) y empatía; siendo la empatía el indicador a tener en consideración para evaluar en la actual investigación, dado que se encuentran las relaciones interpersonales inmersa en la misma, así la empatía desde la visión de Parasuraman, Zeithaml y Berry (2005), como: "atención individualizada que ofrecen las empresas a sus competidores".

Para medir este indicador se toman los cuestionarios propuestos por Parasuraman, Zeithaml y Berry (2005), que permiten calcular las percepciones (Pj) menos las expectativas (Ej) para cada pareja de afirmaciones. De la cual se obtiene la siguiente 
fórmula: SERVQUAL $=\Sigma(P j-E j)$. Con esta comparación se puede obtener tres posibles situaciones:

1. Que las expectativas sean mayores que las percepciones: lo que significa niveles bajos de calidad. (Resultados=-1).

2. Que las expectativas sean menores que las percepciones: los clientes alcanzan niveles altos de calidad y encuentran satisfacción. (Resultados $=1$ )

3. Que las expectativas igualen a las percepciones: entonces los niveles de calidad son pobres. (Resultados $=0$ ).

Así la empatía se promueve como la acción que permite interactuar entre las personas en capacidad de comprender las perspectivas y necesidades de la otra persona, articulándose una relación de servicio basado en el humanismo, así Aldana \& Piña (2018), plantean que la empatía en las relaciones empresariales permite contribuir a la conformación de un servicio de calidad a los usuarios, siendo importante medirla en la gestión pública del derecho, para conocer el comportamiento de esta variable a fin de promover un adecuado ejercicio de las relaciones interpersonales en la administración pública. Por consiguiente, Parasuraman, Zeithaml y Berry (2005), destacan que la empatía permite:

- Atención Individualizada que ofrecen las empresas a los consumidores

- La empresa de servicios da a sus clientes una atención individualizada.

- La empresa de servicios tiene horarios de trabajo convenientes para todos sus clientes.

- La empresa de servicios tiene empleados que ofrecen una atención personalizada a sus clientes.

- La empresa de servicios se preocupa por los mejores intereses de sus clientes.

- La empresa de servicios comprende las necesidades específicas de sus clientes. Las características planteadas se adaptan a la realidad del derecho público como servicio de calidad prestado a las personas, generándose una interrelación que permite conocer 
el grado de empatía promovido, lo cual a partir de Parasuraman, Zeithaml y Berry (2005), se puede calcular a la luz de las tres situaciones planteadas líneas anteriores.

\section{METODOLOGÍA DE LA INVESTIGACIÓN}

El tipo de investigación fue descriptiva, a partir de lo propuesto por Arias (2009), lo cual permitió describir el objeto de estudio a partir de las perspectivas reflejadas por la población encuestada, teniendo como objetivo general: analizar el derecho público como regulador de las relaciones entre las personas. Lo cual permitió realizar un análisis estadístico para describir el comportamiento del derecho público y las relaciones personales de quienes se ven envueltos en el proceso judicial, siendo este concebido como servicio público el cual debe ser ofrecido en calidad al cliente.

Tamayo y Tamayo (2007), destaca que la población "Es la totalidad del fenómeno a estudiar donde las unidades de población poseen una característica común la cual se estudia y da origen a los datos de la investigación" (p. 114), se trabajó en base a 11 personas que recibieron la prestación de servicios relacionados al derecho público en Manta, Ecuador, aplicándosele un instrumento de cinco opciones en escalamiento de Likert, el cual obtuvo un coeficiente de Alfa de Cronbach de 0.89 catalogándose como altamente confiable para su aplicación. 


\section{RESULTADOS}

Los resultados se presentan a la luz de la encuesta aplicada de la adaptación del instrumento de Parasuraman, Zeithaml y Berry (2005), con la finalidad de medir la dimensión empatía, la cual permite establecer las relaciones personales desde un enfoque en donde el servidor público y el cliente, abordan el derecho público.

Atención individualizada

Cuadro 1

\begin{tabular}{|c|c|c|c|c|c|c|c|c|c|c|}
\hline \multirow{2}{*}{ ITEMS } & \multicolumn{2}{|c|}{ SIEMPRE } & \multicolumn{2}{c|}{$\begin{array}{c}\text { CASI } \\
\text { SIEMPRE }\end{array}$} & \multicolumn{2}{c|}{$\begin{array}{c}\text { ALGUNAS } \\
\text { VECES }\end{array}$} & \multicolumn{2}{c|}{$\begin{array}{c}\text { CASI } \\
\text { NUNCA }\end{array}$} & \multicolumn{2}{|c|}{ NUNCA } \\
\cline { 2 - 11 } & FA & $\%$ & FA & $\%$ & FA & $\%$ & FA & $\%$ & FA & $\%$ \\
\hline 1 & 2 & 18 & 3 & 28 & 1 & 9 & 2 & 18 & 3 & 27 \\
\hline 2 & 2 & 18 & 1 & 9 & 3 & 27 & 2 & 18 & 3 & 27 \\
\hline
\end{tabular}

El ítems 1 refleja para la opción siempre una representación estadística del 18\%, casi siempre un $28 \%$, las alternativas algunas veces $9 \%$ y las opciones casi nunca $18 \%$ y nunca el $27 \%$

El ítems 2, refleja para la opción siempre una representación estadística del 18\%, así mismo la opción casi siempre un $9 \%$, las alternativas algunas veces $27 \%$ y las opciones casi nunca $18 \%$ y nunca el $27 \%$

\section{Horario de atención}

Cuadro 2

\begin{tabular}{|c|c|c|c|c|c|c|c|c|c|c|}
\hline \multirow{2}{*}{ ITEMS } & \multicolumn{2}{|c|}{ SIEMPRE } & \multicolumn{2}{c|}{$\begin{array}{c}\text { CASI } \\
\text { SIEMPRE }\end{array}$} & \multicolumn{2}{c|}{$\begin{array}{c}\text { ALGUNAS } \\
\text { VECES }\end{array}$} & \multicolumn{2}{c|}{$\begin{array}{c}\text { CASI } \\
\text { NUNCA }\end{array}$} & \multicolumn{2}{c|}{ NUNCA } \\
\cline { 2 - 11 } & & $\%$ & FA & $\%$ & FA & $\%$ & FA & $\%$ & FA & $\%$ \\
\hline 3 & 1 & 9 & 2 & 18 & 1 & 9 & 2 & 18 & 5 & 46 \\
\hline 4 & 1 & 9 & 1 & 9 & 2 & 18 & 3 & 27 & 4 & 37 \\
\hline
\end{tabular}


El ítems 3, refleja para la opción siempre una representación estadística del 9\%, así mismo la opción casi siempre un $18 \%$, la alternativa algunas veces $9 \%$ y las opciones casi nunca con un $18 \%$ y nunca $46 \%$

El ítems 4, refleja para la opción siempre tuvo una representación estadística del 9\%, así mismo la opción casi siempre un $9 \%$, las alternativas algunas veces $18 \%$ y las opciones casi nunca con un $27 \%$ y nunca con el $37 \%$

Intereses del cliente

Cuadro 3

\begin{tabular}{|c|c|c|c|c|c|c|c|c|c|c|}
\hline \multirow[t]{2}{*}{ ITEMS } & \multicolumn{2}{|c|}{ SIEMPRE } & \multicolumn{2}{|c|}{$\begin{array}{c}\text { CASI } \\
\text { SIEMPRE }\end{array}$} & \multicolumn{2}{|c|}{$\begin{array}{l}\text { ALGUNAS } \\
\text { VECES }\end{array}$} & \multicolumn{2}{|c|}{$\begin{array}{c}\text { CASI } \\
\text { NUNCA }\end{array}$} & \multicolumn{2}{|c|}{ NUNCA } \\
\hline & FA & $\%$ & FA & $\%$ & FA & $\%$ & FA & $\%$ & FA & $\%$ \\
\hline 5 & 1 & 9 & 2 & 18 & 2 & 18 & 4 & 37 & 2 & 18 \\
\hline 6 & 1 & 9 & 2 & 18 & 1 & 9 & 4 & 37 & 3 & 27 \\
\hline
\end{tabular}

El ítems 5, refleja para la opción siempre tuvo una representación estadística del 9\%, así mismo la opción casi siempre un 18\%, la alternativa algunas veces $18 \%$ y las opciones casi nunca $37 \%$ y nunca el $18 \%$

El ítems 6, refleja para la opción siempre tuvo una representación estadística del 9\%, así mismo la opción casi siempre un $18 \%$, las alternativas algunas veces $9 \%$ y las opciones casi nunca con un $37 \%$ y nunca con el $27 \%$

Necesidades específicas de los clientes Cuadro 4

\begin{tabular}{|c|c|c|c|c|c|c|c|c|c|c|}
\hline \multirow{2}{*}{ ITEMS } & \multicolumn{2}{|c|}{ SIEMPRE } & \multicolumn{2}{c|}{$\begin{array}{c}\text { CASI } \\
\text { SIEMPRE }\end{array}$} & \multicolumn{2}{c|}{$\begin{array}{c}\text { ALGUNAS } \\
\text { VECES }\end{array}$} & \multicolumn{2}{c|}{$\begin{array}{c}\text { CASI } \\
\text { NUNCA }\end{array}$} & \multicolumn{2}{c|}{ NUNCA } \\
\cline { 2 - 11 } & FA & $\%$ & FA & $\%$ & FA & $\%$ & FA & $\%$ & FA & $\%$ \\
\hline 7 & 2 & 18 & 1 & 9 & 3 & 27 & 4 & 37 & 1 & 9 \\
\hline 8 & 3 & 28 & 2 & 18 & 3 & 27 & 1 & 9 & 2 & 18 \\
\hline
\end{tabular}


El ítems 7, refleja para la opción siempre tuvo una representación estadística del 18\%, así mismo la opción casi siempre un 9\%, la alternativa algunas veces $27 \%$ y las opciones casi nunca con un $37 \%$ y nunca con un $9 \%$

El ítems 8, refleja para la opción siempre tuvo una representación estadística del 28\%, así mismo la opción casi siempre un $18 \%$, las alternativas algunas veces $27 \%$ y las opciones casi nunca $9 \%$ y nunca con un $18 \%$

\section{Discusión}

A la luz de los resultados, Sánchez Olivencia (2013), destaca la importancia que tiene la empatía para que el servidor público pueda prestar un servicio de calidad al cliente, sin embargo, indican una tendencia negativa en las opciones de repuestas, lo cual deje en evidencia que los usuarios consideran como negativo el servicio recibido desde el punto de vista las relaciones personales, enfocadas desde la empatía, desde ese punto de vista los resultados se contraponen a la teoría, siendo necesario que las instituciones dedicadas al derecho público, asuman esta debilidad para que sea trabajada en aras de mejorar.

\section{CONCLUSIONES}

Partiendo de los presupuestos aristotélicos de causalidad, se evidencia como consecuencia que las personas encuestadas manifiestan inconformidad con el servicio prestado a la luz del trato generado por los servidores relacionados al derecho público, producto de un abordaje personal donde no se considera la empatía como una competencia emocional que debe ser abordada para la consideración del establecimiento de una interrelación basada en el respeto mutuo, produciendo además un manejo intercultural de los escenarios públicos en procura de actuar desde la diversidad social enraizadas en las personas como producto de su interacción social.

Al no efectuarse una empatía que permita el establecimiento de relaciones interpersonales afables, el proceso judicial a la luz del derecho público, se encamina al 
caos, anarquía, por cuanto el principio de las relaciones humanas se basa en el respeto reciproco, confrontándose mediante el intercambio de ideas y opiniones, las cuales deben ser conducidas en dialéctica para generar una perspectiva cordial en el trato generado entre las partes involucradas, siendo que esto promueve el cumplimiento de los derechos humanos de las personas como principio de establecimiento de una sociedad basada en el dialogo y en la paz.

\section{REFERENCIAS CONSULTADAS}

1. Arias, F. (2009). El Proyecto de Investigación. Guía para su elaboración. Caracas: Epísteme. Quinta Edición.

2. Aldana, J., \& Piña, J. (2018). Calidad del servicio prestado al cliente por los instructores de gimnasios. Revista Arbitrada Interdisciplinaria Koinonía, 2(3), 172 197. Recuperado de http://fundacionkoinonia.com.ve/ojs/index.php/revistakoinonia/article/view/59/46

3. Borja Cevallos, R. (2019). Derecho público. Recuperado de https://www.derechoecuador.com/derecho-publico

4. Parasuraman, Zeithaml y Berry (2005). A Conceptual Model of Service Quality and Its Implications for Future Research. Journal Of Marketing. Vol. 49.

5. Sánchez Olivencia, C. (2013). El gestor público con empatía es clave para el Estado. Recuperado de http://www.generaccion.com/noticia/187828/gestorpublico-con-empatia-clave-estado

6. Tamayo y Tamayo (2009). El proceso de investigación científica. Limusa. México.

\section{REFERENCES CONSULTED}

1. Arias, F. (2009). The Research Project Guide for its elaboration. Caracas: Epistle me. Fifth edition.

2. Aldana, J., \& Piña, J. (2018). Quality of service provided to the client by gym instructors. Interdisciplinary Arbitrated Review Koinonía, 2 (3), 172-197. Recovered from http://fundacionkoinonia.com.ve/ojs/index.php/revistakoinonia/article/view/59/46 
Edison Aroldo Gracia Panta; Liliana Hipatía Rodríguez Mera; Klevis Gabriel Gracia Panta

3. Borja Cevallos, R. (2019). Public Law. Recovered from https://www.derechoecuador.com/derecho-publico

4. Parasuraman, Zeithaml and Berry (2005). A Conceptual Model of Service Quality and Its Implications for Future Research. Journal of Marketing Vol. 49.

5. Sánchez Olivencia, C. (2013). The public manager with empathy is key to the State. Recovered from http://www.generaccion.com/noticia/187828/gestor-publico-conempatia-clave-estado

6. Tamayo and Tamayo (2009). The process of scientific research. Limusa Mexico. 